\title{
Die Patientensicherheit in der Schweiz
}

Norbert Rosea,

Maria-Anna J. Ortner ${ }^{b}$,

Christa Meyenbergerc,

André L. Blum ${ }^{d}$

a Abteilung Qualitätsmanagement, Kantonsspital St. Gallen

b Gastroenterologie, Inselspital Bern

c Gastroenterologie/ Hepatologie, Kantonsspital St. Gallen

d Faculté de Biologie et Médecine, Lausanne
Das Autorenteam bedankt sich bei den Experten für die Teilnahme (s. Namensliste unter www.saez.ch) und bei der Stiftung Patientensicherheit, für die Benutzung der Namensliste der Referenten der Tagung «Patientensicherheit Schweiz 2007».

* Die Literaturangaben finden sich im Internet unter www. saez.ch $\rightarrow$ aktuelle Ausgabe oder $\rightarrow$ Archiv $\rightarrow 2009 \rightarrow 48$

Korrespondenz: Dr. med. Norbert Rose Leiter Abteilung Qualitätsmanagement Kantonsspital St.Gallen CH-9007 St.Gallen

norbert.rose@kssg.ch

www.kssg.ch/home.htm

\section{Einleitung}

Die Patientensicherheit in den Vereinigten Staaten wurde vor zehn Jahren vom renommierten Institute of Medicine als katastrophal bezeichnet [1]*. Der Schweiz wird im internationalen Vergleich ein relativ gutes Zeugnis ausgestellt [2]; aufgrund unserer eigenen Erfahrung mit Schadenfällen und Meldesystemen für Beinaheschäden (CIRS, Critical Incidence Reporting System) [3] ist die schweizerische Patientensicherheit allerdings stark verbesserungsbedürftig. Zur Klärung der Frage, wie die Experten unsere Patientensicherheit einstufen und welche Verbesserungsmassnahmen sie vorschlagen, haben wir eine Umfrage durchgeführt.

\section{Methoden}

Angeschrieben wurden 78 Experten des Gesundheitswesens, die anlässlich der Tagung «Patientensicherheit Schweiz 2007» als Referenten aufgetreten waren und/oder sich öffentlich zu diesem Thema geäussert hatten. 23 von ihnen hatten täglichen und direkten klinischen Patientenkontakt (Ärzte, Pflegefachpersonen, Physiotherapeuten); 55 befassten sich mit dem Gesundheitssektor ohne klinischen Patientenkontakt. Die Fragebogen wurden zusammen mit einem Informationsbrief zum Hintergrund der Befragung am 4.4. 2008 versandt. Die Antworten konnten per Post, Fax oder Mail eingereicht werden. Eine Nachfassaktion

\section{La sécurité des patients en Suisse}

Lors d'une enquête ( $76 \%$ de réponses), 59 experts du système de santé ont estimé que la sécurité des patients était moyenne à bonne, voire très bonne en Suisse. Les experts en contact régulier avec des patients étaient plus positifs que ceux qui n'en avaient pas; parmi ces derniers, seuls deux ( $4 \%$ des participants à l'enquête) ont estimé que la sécurité des patients était mauvaise à très mauvaise. La question relative aux trois mesures d'amélioration les plus utiles a recueilli 161 propositions qui peuvent se répartir en 21 catégories. Les mesures les plus fréquemment citées concernent la prescription électronique de médicaments (computerized physician order entry), le système de déclaration anonyme d'incidents critiques (critical incidence reporting system) ainsi que la formation prégraduée, postgraduée et continue complétée d'une méthodique propre à améliorer la sécurité des patients.

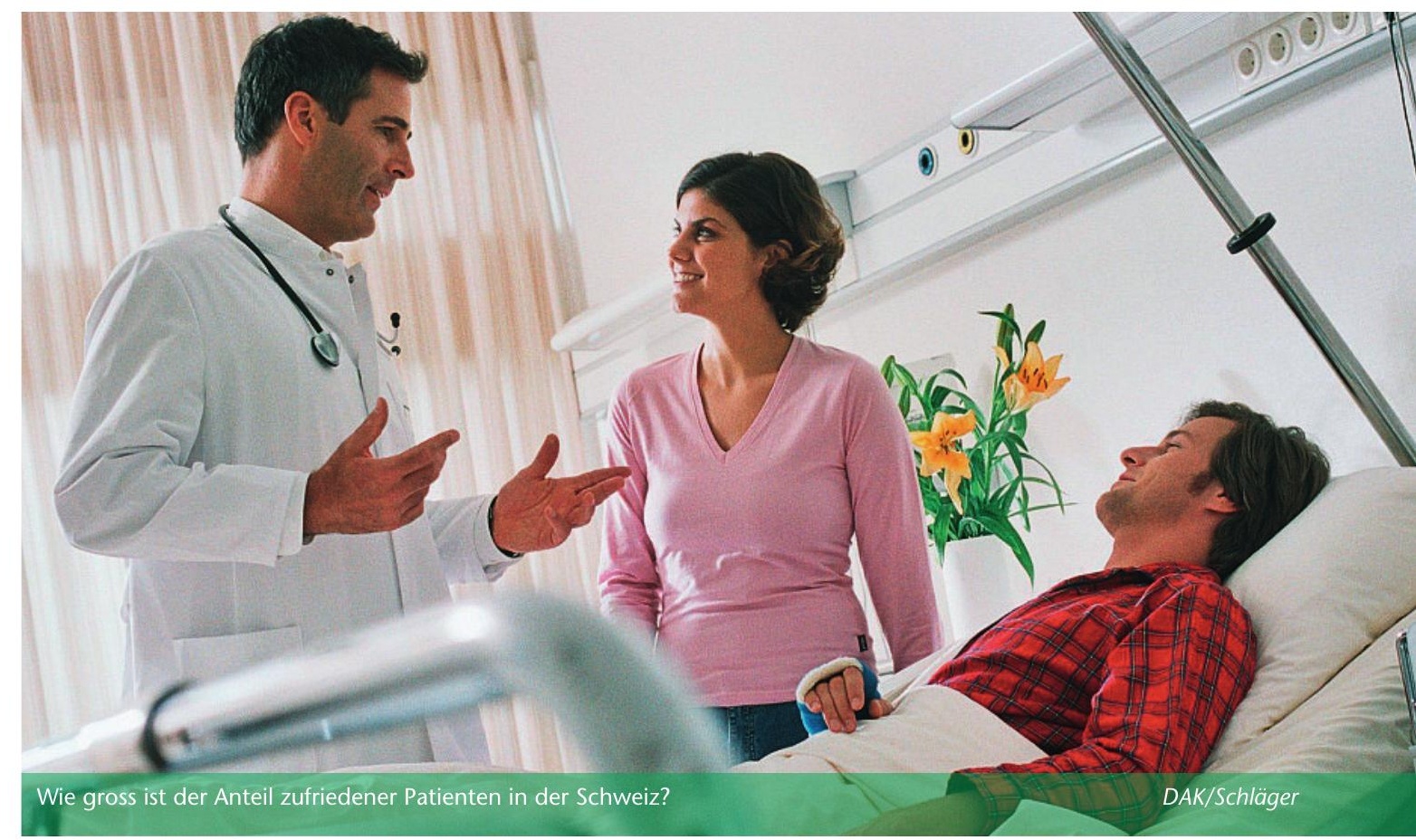


mit einem Erinnerungsbrief wurde am 25.8.2008 gestartet. Am 30.11.2008 wurde die Befragung abgeschlossen.

\section{Abbildung 1}

Antwortverteilung zu Frage 1 «Zufriedenheit mit der Patientensicherheit in Schweizer Institutionen des Gesundheitswesens».

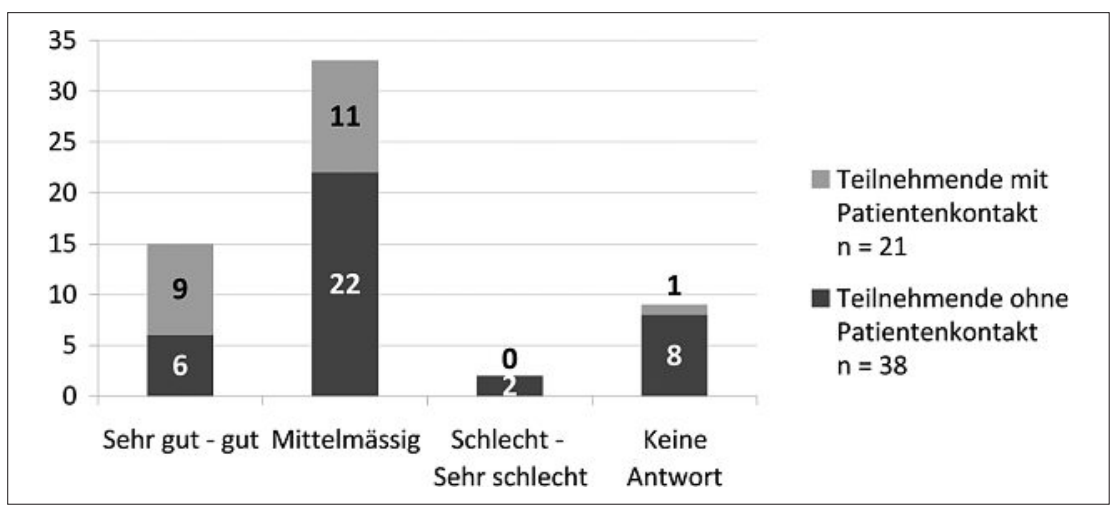

\section{Tabelle 1}

Antwortverteilung zu Frage 2 «Welche drei Massnahmen zur Verbesserung der Patientensicherheit in der Schweiz sind Ihrer Meinung nach die nützlichsten?»

\section{Die nützlichsten Massnahmen zur Verbesserung}

Anzahl der Nennungen

der Patientensicherheit

Medikamentenmanagement verbessern, z.B. CPOE* einführen

CIRS* einführen, CIRS*-Daten schützen

18

18

Aus-, Fort- und Weiterbildung im Bereich der Patientensicherheit verbessern

Fehlerkultur verbessern

Zentralisierungsmassnahmen von Patientensicherheits- und

Qualitätsstrategien auf nationaler Ebene, z.B. CIRRNET* einführen

Hygienemassnahmen verbessern

Standards, Prozesse und klinische Behandlungspfade festlegen

Ergebnisqualität messen und publizieren

Patientenidentifikation verbessern, z.B. Time-out-Verfahren,

Wrong-site-surgery-Massnahmen RFID*

Qualitätsmanagement verbessern, Zertifizierungen durchführen

7

Arbeitsbedingungen verbessern, z. B. Personalengpässe vermeiden

Risikomanagement einführen

Vereinheitlichungen und Definitionen zur Patientensicherheit einführen

Evidenzbasierte Medizin, Kosten-Nutzen-Analysen,

Wirtschaftlichkeitskriterien anwenden

Informationstechnologie einführen,

z. B. elektronische Patientenakte

Sturzprävention verbessern

Patientenpartizipation unterstützen

Haftpflichtdaten öffnen, Schadenmeldesysteme

Sanktionen und Bestrafung der Fehlbaren einführen

DRG*-System nicht einführen

Präventivmedizinische Massnahmen,

z. B. kardiale Reihenuntersuchungen durchführen

Personal der Administration reduzieren

\section{7}

6

6

4

4

4

3

2

2

1

1

* Abkürzungen: CIRRNET = Critical Incidence Reporting and Reacting Network,

$\mathrm{CIRS}=$ Critical Incidence Reporting System, CPOE = Computerized Physician Order Entry,

DRG = Diagnosis Related Groups, RFID = Radio Frequency Identification
Frage 1 lautete: «Sind Sie mit der Sicherheit der Patienten in Schweizer Gesundheitsinstitutionen (Spitälern, Geriatrien und Rehabilitationskliniken) zufrieden?» Die Antwortkategorien waren: Die Sicherheit ist: «gut bis sehr gut», «mittelmässig», «schlecht bis sehr schlecht».

Frage 2 lautete: «Welche drei Massnahmen zur Verbesserung der Patientensicherheit in der Schweiz sind Ihrer Meinung nach die nützlichsten?» Wir ordneten die Freitextantworten 21 Massnahmenkategorien (s. Tab. 1) zu.

\section{Resultate}

Die Rücklaufquote betrug 76\%. Die Experten mit regelmässigem Patientenkontakt zeigten eine grössere Bereitwilligkeit, an der Befragung teilzunehmen $(21 / 23=91 \%)$ als jene ohne einen solchen Kontakt $(38 / 55=69 \%$, Fisher's Exakt p = 0,045).

Zu Frage 1 nahmen 50 der 59 Teilnehmer (20 mit und 30 ohne Patientenkontakt) Stellung (siehe Abb. 1). 6 Teilnehmer gaben an, die Frage nicht beantworten zu können, 3 Teilnehmer liessen das Antwortfeld ohne Begründung frei. $43 \%$ der Teilnehmer mit und 16\% der Teilnehmer ohne Patientenkontakt bezeichneten die Patientensicherheit als gut bis sehr gut (Fisher's Exakt $\mathrm{p}=0,031)$. Nur zwei (4\%) Teilnehmer, beide ohne direkten Patientenkontakt, bezeichneten die Patientensicherheit als schlecht bis sehr schlecht.

Frage 2 wurde von 54 Teilnehmern beantwortet. 5 Teilnehmer fühlten sich ausserstande, eine Antwort im Sinn der Umfrage zu geben. Ein Teilnehmer nannte nur zwei Massnahmen. Tabelle 1 zeigt die zugeordneten 161 Vorschläge.

\section{Diskussion}

Der Patientensicherheit in der Schweiz wird von einer Gruppe von Experten kein allzu schlechtes Zeugnis ausgestellt. Den Befragten ist gemeinsam, dass sie an einer Tagung über Patientensicherheit im Jahre 2007 als Referenten teilgenommen und/oder sich öffentlich zur Patientensicherheit geäussert haben. Aufgrund der an der Tagung vorherrschenden Besorgnis erwarteten wir, dass die Patientensicherheit in der Schweiz als schlecht eingestuft würde und waren überrascht über das eher günstige Bild. Die Schweizer Patientensicherheit schneidet im europäischen Vergleich recht gut ab [2]. Mit den Antworten auf unsere Frage «Sind Sie mit der Sicherheit der Patienten in Schweizer Gesundheitsinstitutionen zufrieden?» können wir allerdings keine Aussagen über den tatsächlichen Zustand der Patientensicherheit machen. Von einer Meinungsabfrage bis zur Erhebung von Fakten liegt ein weiter Weg.

Weshalb steht von allen Facetten der Behandlungsqualität gerade die Patientensicherheit im Zentrum des Interesses? Da sie in der Schweiz - im Gegensatz vielleicht zu anderen Ländern [1, 2] - nicht aus dem qualitativen Rahmen fällt, müssen andere Gründe gesucht werden. Behandlungsqualität ist für viele ein 
praxisferner Begriff, während die Untergruppe der Patientensicherheit jedes Mitglied des Behandlungsteams und letztlich jeden Bürger anspricht. Aus der Patientensicherheit lässt sich auch politisches Kapital schlagen. Es besteht dabei die Gefahr, dass als Folge der Überbetonung von Sicherheitsaspekten andere Qualitätsprobleme zu kurz kommen. Allerdings zeigen die 161 Vorschläge der Experten, dass bei einer Verbesserung der Sicherheit der gesamte Qualitätsstandard angehoben wird. solche Lehrtätigkeit [7] und hat einen Lernzielkatalog erstellt, der dem heute schon sehr vollen medizinischen Curriculum zugefügt werden soll.

An zweiter Stelle (14 Nennungen) werden ex aequo «Fehlerkultur» und «Zentralisierungsmassnahmen» genannt. Als Fehlerkultur wird die Bereitschaft der Mitarbeitenden eines Teams verstanden, Fehler nicht zu vertuschen, sondern dem Team mitzuteilen und Kritik als Aufforderung zur Problemlösung aufzufassen. Die Sicherheit der Patienten soll höher ein-

\section{Wir empfehlen, die Patientensicherheit nur mit ethisch vertretbaren, kostenwirksamen Massnahmen zu verbessern und keinem gesundheits- politisch motivierten Aktivismus zu verfallen}

Wir fragten nicht nach den besten, sondern nach den nützlichsten Massnahmen. Der Nutzen hängt von der Häufigkeit des Problems im eigenen Land, den zur Verfügung stehenden Mitteln, den geleisteten Vorarbeiten, der Durchführbarkeit von Massnahmen und vor allem von Kosten-Nutzen-Berechnungen ab. Sind elektronische Radiofrequenz-Identifikationssysteme zur Patientenidentifikation - um nur ein Beispiel zu nennen - nützlich? Wie viele Mittel bleiben zur Lösung anderer Sicherheitsprobleme und zur generellen Verbesserung der Qualität übrig, wenn sich ein Spital zugunsten dieser zwar wirksamen, aber teuren Technologie entscheidet? Diese Überlegungen sind bei der Diskussion der «nützlichsten» Massnahmen zu berücksichtigen.

Die drei häufigsten Nennungen durch die Experten $(\mathrm{n}=18)$ betreffen «Medikamentenmanagement», «Critical Incidence Reporting System» (CIRS) und «Aus-, Fort- und Weiterbildung». Mindestens die Hälfte aller vermeidbaren Behandlungsfehler wird durch Medikamentenfehler verursacht [4]. Entsprechend wird eine elektronische Arzneimittelverschreibung mit automatischen Hinweisen auf mögliche Arzneimittelinteraktionen gefordert. Diese Massnahme war ein Teil der erfolgreichen «100000-Leben-Kampagne» [5]. Das CIRS, ein anonymes Meldesystem für Beinaheschäden, beruht auf der Beobachtung, dass die gleichen Fehlerschritte einmal zu Beinaheschäden, unter anderen Umständen dagegen zu Schäden führen. Durch kontinuierliche Massnahmen zur Beseitigung der Fehlerschritte sollten sich in Zukunft Schäden verhindern lassen. Leider fehlt in der Schweiz die für das CIRS nötige gesetzliche Grundlage [6]. Zudem lässt sich heute in Ermangelung von einheitlichen Definitionen zur Patientensicherheit der mögliche Nutzen des CIRS nicht adäquat messen. Die Aus-, Fort- und Weiterbildung gründet sich auf der - noch zu beweisenden Annahme, dass die sogenannte Fehlerkultur lehr- und lernbar ist. Die Schweizerische Akademie der Medizinischen Wissenschaften legt grossen Wert auf eine gestuft werden als die egoistischen Interessen. Wir konnten mittels einer Mitarbeiterbefragung [3] nachweisen, dass sich die Fehlerkultur durch ein CIRS verbessern lässt. Eine mögliche Zentralisierungsmassnahme ist der Aufbau eines nationalen CIRS (CIRRNET) [8]. Allerdings fehlt hier eine Kosten-Nutzen-Analyse. Die staatliche Förderung von Zentren mit grosser spezifischer Erfahrung zur Verbesserung der Patientensicherheit wird ebenfalls kontrovers diskutiert [9]. Offenbar ist es in grossen Systemen schwieriger als in kleinen Einheiten, die Sicherheit des einzelnen Patienten im Auge zu behalten; «grösser» ist nicht automatisch «besser». Die Führung von nationalen OutcomeRegistern wird unten diskutiert. Gewarnt wird vor der blinden Übernahme nordamerikanischer Zentralisierungsmodelle [1, 2, 4].

Mit «Hygienemassnahmen» (Platz 3, 11 Nennungen) werden nosokomiale Infektionen, insbesondere mit multiresistenten Spitalkeimen, vermieden. In der «100000-Leben-Kampagne» [5] lag das Schwergewicht auf zentralen Kathetern, Operationswunden und Beatmungsinfekten. Der organisatorische und personelle Aufwand dieser ergebniswirksamen Massnahmen ist beträchtlich.

Auf Platz 4 (10 Nennungen) liegen «Standards, Prozesse, klinische Behandlungspfade» sowie «Ergebnisqualität». Behandlungspfade, basierend auf Leit- und Richtlinien, standardisieren die diagnostischen, therapeutischen und pflegerischen Massnahmen, speziell während der Hospitalisation. In Bezug auf Kosteneinsparungen sind die Akten nicht geschlossen. Für die Transparenz der Ergebnisqualität entscheidend ist die Outcome-Messung durch spitalinterne Register. Der Nutzen ihrer Publikation ist nicht gesichert [10]. Die vom Bundesamt für Gesundheit publizierten Mortalitätsstatistiken haben in der Schweiz eine heftige Kontroverse zu Grenzen und Sinnhaftigkeit von Vergleichsdaten (Benchmarking) verursacht. Prospektive Studien mit einheitlichem Protokoll sind eine selten gewählte, teure und arbeitsintensive Maximallösung. 
Platz 5 (7 Nennungen) nehmen «Patientenidentifikation» sowie «Qualitätsmanagement, Zertifizierungen» ein. Fehler der Patientenidentifikation sind auch in Schweizer Spitälern häufig. Durch einfache, direkt vor Operationsbeginn durchzuarbeitende Checklisten [Time-out-Verfahren, 11] wird eine kostengünstige Senkung von Komplikationen und Todesfällen erreicht. Die Zertifizierung der Qualitätsstandards sollte auch die Patientensicherheit verbessern, doch ist die Diskussion zu dieser Frage nicht abgeschlossen [12]. Allgemeine Qualitätsaspekte haben wir eingangs besprochen.

Auf Platz 6 (6 Nennungen) stehen «Arbeitsbedingungen» und «Risikomanagement». Gute Arbeitsbedingungen, Sicherheit und Gesundheit des Handlungsteams, ausreichende Personaldotierung, Kompensation der Überzeit und gerechte Saläre wirken sich wahrscheinlich günstig auf Faktoren wie die Verhütung nosokomialer Infektionen aus [13]. Beim Risikomanagement wird das traditionelle, kontinuierliche Erkennen, Analysieren und Verhüten von Risiken und Fehlern professionalisiert und zentralisiert. In einer schweizerischen Erhebung [14] wird diese administrative Massnahme empfohlen. Ihre Kosteneffektivität ist allerdings nicht erwiesen.

Nicht besonders populär (Platz 7; 4 Nennungen) sind «Vereinheitlichungen, Definitionen» und «Evidenzbasierte Medizin, Kosten-Nutzen-Analysen, Wirtschaftlichkeit» und «Informationstechnologie». Wir mussten leider feststellen [15], dass im Bereich der Patientensicherheit bis heute einheitliche Definitionen selbst der einfachsten Begriffe fehlen. Somit ist es nicht klar, was verbessert werden soll, wie Verbesserungen gemessen und wie sie kommuniziert werden können. Verwertbare Grundlagen für einen Schweizer Definitionskatalog wären vorhanden [16]. Die evidenten Massnahmen zur Verbesserung der Patientensicherheit sind in einer Metaanalyse beschrieben worden [17]. Allerdings spielen neben der statistischen Evidenz die Begleitumstände eine entscheidende Rolle, beispielsweise technische Fertigkeiten und Patientenselektion; zudem übersteigen die Kosten oft den Nutzen. Leider gibt es keine «richtige» Art der Kostenberechnung [18]; jede Interessensgruppe kommt auf andere Zahlen. In der Schweiz erarbeitet die Arbeitsgruppe Qualität der FMH [19] «WZW-Kriterien» für W(irksamkeit), Z(weckmässigkeit) und W(irtschaftlichkeit). Allerdings sollten vor deren Anwendung ethischen Grundlagen erarbeitet werden [20], und es gilt zu verhüten, dass durch die schleichende Rationierung der Leistungen die Patientensicherheit verschlechtert wird [21]. eHealth umschreibt die Informatik und Kommunikationstechnologie im Gesundheitswesen, deren Einführung in der Schweiz schon längst begonnen hat. In welchem Ausmass dadurch - jenseits des oben diskutierten Medikamentenmanagements - kostenwirksam die Kommunikation beschleunigt, die Transparenz und die Patientensicherheit verbessert wird, muss erst noch gezeigt werden.

Selten genannt (3 Nennungen) wird die Sturzprävention; für spitalumfassende Programme ist hier die Evidenz spärlich [21]. Die Patientenpartizipation (2 Nennungen) setzt voraus, dass die Patienten aus ihrer traditionell passiven Rolle heraustreten [23] die Auswirkungen auf die Sicherheit können je nach den Umständen günstig oder schädlich sein. Schadenmeldesysteme (2 Nennungen) benötigen eingreifende flankierende juristisch-administrative Massnahmen, wobei der Nutzen insgesamt fraglich bleibt. Die 1erNennungen werden hier nicht weiter diskutiert.

\section{Drei eigene Massnahmenvorschläge}

Wir selbst erachten drei Massnahmen als besonders wichtig. Erstens plädieren wir zugunsten von Vereinheitlichungen und verbindlichen Definitionen. Wenn die Auswirkungen der Massnahmen zur Verbesserung der Patientensicherheit nicht messbar sind, lässt sich ihre Kostenwirksamkeit nicht quantifizieren, und damit bleibt die Sicherheitspolitik ein Spielball von Meinungen und Modeströmungen. Die zweite von uns bevorzugte Massnahme ist trotz der oben erwähnten Nachteile das CIRS. Wir haben seit seiner Einführung eine Verbesserung der «Fehlerkultur» beobachtet, die sich in einer wachsenden Zahl von Meldungen und Behebung von Problemen äussert [3]. Allerdings ist dies nur ein indirekter Hinweis auf den Nutzen; zur quantitativen Erfolgsmessung und Kosten-NutzenAnalyse stehen uns keine Instrumente zur Verfügung stehen. Wir warten auf Studien, die zeigen, dass ein selbstgeschneidertes Massnahmenpaket mehr bringt als ein einheitliches, wie es beispielsweise in der 100000 -Leben-Kampagne verwendet worden ist [5]. Die dritte uns wichtig erscheinende Massnahme, nämlich die Ausarbeitung ethischer Grundlagen, ist von keinem der Experten genannt worden. Das erstaunt uns, denn der ethische Imperativ aus den Anfängen der Medizin, in erster Linie dem Patienten nicht zu schaden, ist mit neuen Massnahmen, die das Qualitätsmanagement und die Patientensicherheit nachhaltig beeinflussen werden, nämlich dem System der Fallpauschalen (DRG) [20], den WZW-Kriterien [19] und der sich daraus ergebenden Rationierung medizinischer Leistungen [21] nicht vereinbar. Wir empfehlen daher, die Patientensicherheit nur mit ethisch vertretbaren, kostenwirksamen Massnahmen $\mathrm{zu}$ verbessern und dabei keinem gesundheitspolitisch motivierten Aktivismus zu verfallen. 


\title{
Literaturliste mit zusätzlichen Literaturstellen zum Artikel „Die Patientensicherheit in der Schweiz, Resultate einer \\ Expertenbefragung", Schweizerische Ärztezeitung,2009;90: 48 \\ (Redaktionelle Verantwortung bei den Autoren)
}

\author{
Autoren \\ Faculté de Biologie et Médecine, Lausanne \\ Korrespondenzadresse: \\ Dr. med. Norbert Rose \\ Leiter Abteilung Qualitätsmanagement \\ Kantonsspital St.Gallen \\ $\mathrm{CH}-9007$ St.Gallen
}

Norbert Rose ${ }^{1}$, Maria-Anna J. Ortner ${ }^{2}$, Christa Meyenberger ${ }^{3}$ und André L. Blum ${ }^{4}$

1: Abteilung Qualitätsmanagement, Kantonsspital St.Gallen; 2: Gastroenterologie, Inselspital Bern; 3: Gastroenterologie/Hepatologie, Kantonsspital St.Gallen; 4:

\section{Literaturstellen zum Artikel in der SÄZ, Rose N. „Die Patientensicherheit in der} Schweiz", Schweizerische Ärztezeitung,2009;90: 48

1 Kohn LT, Corrigan JM, Donaldson MS Hsg. To Err Is Human: Building a Safer Health System. National Academy Press, Washington 2000. http://books.nap.edu/catalog/9728.html\#toc

2 Erikson D., Bjornberg A,The Euro-Canada Health Consumer Index 2009. FCPP Policy Series No.61

http://www.healthpowerhouse.com/index.phpoption=com content\&view=article\&id=5 $\underline{5 \& \text { ltemid }=54}$

3 Rose N., Hess U. Melden von Near Misses im Krankenhaus; Der Onkologe 2008 DOI 10.1007/s00761-008-1402-5

4 Aspden P, WolcottJA, Bootman JL et al, Hsg. Preventing Medication Errors. National Academic Press Washington DC 2007

5 McCannon CJ, Schall MW, Calkins DR, Nazem AG. Saving 100000 lives in US hospitals. BMJ 2006;332:1328

6 Kuhn H. 10 Jahre Warten auf gesetzliche Zweckbindungsgarantie für CIRS. SÄZ 2009; 90:95

7 Perrier A und Arbeitsgruppe SAMW. Aus- und Weiterbildung in Patientensicherheit und Sicherheitskultur; Projekt "Zukunft der Medizin Schweiz", Phase III. Schweizerische Akademie der Medizinischen Wissenschaften 2007, http://www.samw.ch

8 http://www.cirrnet.ch/ local/cirrnet homepage/read write/public/12430.pdf 
9 Lindenauer PK, Rothberg MB, Pekow PS, Kenwood C, Benjamin EM, Auerbach A D. Outcomes of care by hospitalists, general internists and family physicians. N Engl J Med 2007; 357:2589-600

10 Fung $\mathrm{CH}$, Lim Y-W, Mattke S, Damberg C, Shekelle PG. Systematic review: the evidence that publishing patient care perfomance data improves quality of care. Ann Intern Med 2008; 148:111-123

11 Haynes AlB., Weiser TG., Berry WR und Safe Surgery Saves Lives Study Group. A surgical safety checklist to reduce morbidity and mortality in a global population. $\mathrm{N}$ Engl J Med 2009; 360:491-9

12 Snyder C, Anderson G. Do quality improvement organizations improve the quality of hospital care for Medicare beneficiaries? JAMA 2005, 293:2900-7

13 Virtanen M, Kurvinen T, Terho K, Oksanen T, Peltonen R, Vahtera J, Routamaa M, Elovainio M, Kivimäki M. Work hours, work stress, and collaboration among ward staff in relation to risk of hospital-associated infection among patients. Med Care 2009; 47:310-8

14 Briner M., Kessler O, Pfeiffer Y., Wehner T., Manser T. Erste Schweizer Erhebung zum klinischen Risikomanagement im Spital. SÄZ 2009;90:15/16

15 Meyenberger C, Ortner M, Blum AL. Der medizinische Irrtum. Vom menschlichen Versagen zum Fehler im System. In: Ingold FP und Sanchez Y Hsg. Fehler im System. Wallstein Göttingen 2008, S. 91- 109

16 Chang A, Schyve PM, Croteau RJ, O'Leary DS, Loeb JM. The JCAHO patient safety event taxonomy: a standardized terminology and classification schema for near misses and adverse events. Int J Qual Health C 2005;17: 95-1005

17 Shojana KG, Duncan BW, McDonald KM et al. Safe but sound. Patent safety meets evidence-based medicine. J Am Med Assoc 288:508-513, 2002; http://www.NOF.org.

18 Kirschner N, Pauker SG, Stubbs JW und American College of Physicians. Information on cost-effectiveness: an essential product of a national comparative effectiveness program. Ann Int Med 2008 ;148:956-961

19 Arbeitsgruppe Qualität der FMH. Wirksam, zweckmässig, wirtschaftlich - Betrachung aus ärztlicher Sicht. Positionspapier und Checkliste zur Zweckmässigkeit. SÄZ 2007; 88:1665-9

20 Wild V, Biller-Adorno N. Einführung der Fallpauschalen in der Schweiz - eine Frage der Ethik. SÄZ 2008: 89:361-366

21 Paccaud F und Arbeitsgruppe <Rationierung > der SAMW. Rationierung im Schweizer Gesundheitswesen: Einschätzung und Empfehlung. SÄZ 2007; 888: 1431-7

22 Schwendimann René, Bühler Hugo, De Geest Sabina and Milisen Koen. Falls and consequent injuries in hospitalized patients: effects of an interdisciplinary falls prevention program. BMC Health Services Research 2006,6:69 doi:10.1186/1472- 
6963-6-69

23 Schwappach DLB. „Against the silience“: Development and first results of a patient survey to assess experiences of safety-related events in hospital. BMC Health Services Research 2008:8; http://biomedcentral.com/1472-6963/8/59

\section{Zusätzliche Literaturstellen zum Artikel in der SÄZ, Rose N. „Die Patientensicherheit in der Schweiz"}

24 Giger M. Ärztliche Berufsbildung und Patientensicherheit. SÄZ 2007; 88: 1239

25 Takata GS, Takemoto CK, Waite S. Characteristics of medication errors and adverse drug events in hospitals participating in the California Pediatric Patient Safety Initiative. Am J Health-Syst Pharm 2008; 65:2036-2044

26 Simpson ChM, Keijzers GB,Lind JF. A survey of drug-dose calculation skills of Australian tertiary hospital doctors. Medical Journal of Australia 2009; 190:117-120.

27 Rose N., Germann D. Resultate eines krankenhausweiten Critical Incident Reporting System. Das St. Galler CIRS-Konzept. Gesundh ökon manag; 10:83-89.

$28 \mathrm{http}: / /$ www.hadmedical.ch/had.htm

29 Lovis Ch, Bondolfi A, Bellucci S. eHealth en Suisse: chances et risques. Bulletin SAMW 3/08, 2008.

30 Hodgson CS. Teherani A, Gough HG, Bradley P, Papadakis MA. The relationship between measures of unprofessional behavior during medical school and indices on the California Psychological Inventory. Academic Medicine 2007; Suppl 82:S4-S7

31 Bosley S, Dale J. Healthcare assistants in general practice: practical and conceptual issues of skill-mix change. British Journal of General Practice 2008; 58:118-124

32 Stryer D, Clancy C. Patients' safety. BMJ 2005; 553-4, 2005

33 Bachstein S. Über Medizinfehler sprechen und daraus lernen. SÄZ 2008; 89, 448

34 Mazzocco K, Petitti DB, Fong KT, Bonacum D, Brookey J, Graham S, Lasky RE, Sexton JB, Thomas EJ. Surgical team behaviors and patient outcomes. American Journal of Surgery 2008, Epub ahead of publication.

35 Walter Andreas. Das Schweizer Gesundheitswesen ist ein System. SÄZ 2008;89:704-706

36 http://www.cirrnet.ch/index.asp

37 Bundesamt für Gesundheit BAG. Qualitätsindikatoren der Schweizer Akutspitäler: Spezifikation V1.0. www.bag.ch/kvstat

38 Baker GR, Norton PG, Flintoft V, Blais R, Brown A, Cox J, Etchells E, Ghali WA, 
Hébert P, Majumdar SR, O'Beirne M, Palacios-Derflingher L, Reid RJ, Sheps S, Tamblyn R. The Canadian Adverse Event Study: the incidence of adverse events among hospital patients in Canada. JAMC 2004;170:1678-86

39 Flanders SA., Collard HR, Saint S. Nosocomial pneumonia: State of the science. Am J Infect Control 2006; 34:84-93

40 March LM, Cameron ID, Cummings RG, et al. Mortality and morbidity after hip fracture: can evidence based clinical pathways make a difference? J Rheumatol. 2000; 27:2227-2231

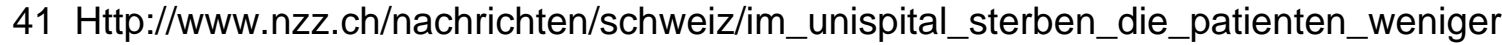
_oft_1.1760538.html

42 Percarpio KB, Watts BV, Weeks WB. The effectiveness of root cause analysis; what does the literature tell us? Jt Comm J Qual Patient Saf 2008; 34:391-8

43 Vincent Ch, Taylor-Adams S, Chapman EJ, Hewett D, Prior S, Strange S, Tiizzard A. How to investigate and analyse clinical incidents: Clinical Risk Unit and Association of Litigation and Risk Management protocol. BMJ 2000; 320:777-81

44 Berwick DM. Taming the technology beast. JAMA 2008; 299:2898-2899

45 Kahla-Witzsch HA. Praxis des Klinischen Risikomanagement, Ecomed Medizin 2005 S.15

46 www.who.int/entity/patientsafety/taxonomy/Final_Report_of_Drafting_Group05.pdf

47 Runciman W, Hibbert P, Thomson R, Van Der Schaaf Tj, Sherman H, Lewalle P. Towards an International Classification for Patient Safety: key concepts and terms. Int J Qual Health Care. 2009; 21:18-26.

48 Brauer S und die Nationale Ethikkommission. Zur Einführung von diagnosebezogenen Fallpauschalen in Schweizer Spitälern. Stellungnahme Nr. 15/2008 der NEK-CNE. SÄZ 2008;89:1533-6

49 Krumholz H. Outcomes Reserach. Generating evidence for the best practice and policies. Circulation 2008: 118:309-318

50 Cabana MD, Rand CS, Powe NR et al.: Why don't physicians follow clinical practice guidelines? A framework of improvement. Journal of the American Medical Association 1999; 282:1458-1465

51 Giger Max. Workshop HPC und E-Health. SÄZ 2008; 89:1701-6

52 Bjornberg A, Uhlir M. The Euro Health Consumer Index 2008. http://www.healthpowerhouse.com/index.phpoption=com_content\&view=article\&id=5 $5 \&$ Itemid $=54$

53 bto. Spitäler sündigen beim Patientengeheimnis. NZZ 2009; 9.März,S.47

54 Qualitätsbericht 2008 Kantonsspital St.Gallen, www.kssg.ch 
55 Oliver D, Connelly JB, Victor CR, Shaw FE, Whitehead A, Genc Y, et al. Strategies to prevent falls and fractures in hospitals and care homes and effect of cognitive impairment: systematic review and meta-analyses. BMJ 2007 Jan 13;334(7584):82. Epub 2006 Dec 8. Review.

56 Coulter A, Ellins J. Effectiveness of strategies for informing, educating and involving patients. BMJ 2007;335:24-27

57 Snijders C, van Lingen RA, Klip H, Fetter WPF, van der Schaaf TW Molendijk HA. Specialty-based, voluntary incident reporting in neonatal intensive care: description of 4846 incident reports.Arch. Dis. Child. Fetal Neonatal Ed. published online 6 Oct 2008;doi:10.1136/adc.2007.135020

58 Youngberg BJ. Event reporting: the values of a nonpunitive approach. Clinical Ostetrics and Gynecology 2008: 51:647-655

59 http://www.bag.admin.ch/nek-cne/06238/index.html?lang=de

60 hof. Jeder ein Philosophenkönig. Wieso Ethikkommissionen nicht über moralische Autorität verfügen.NZZ 2009; 16. April, S. 16 


\section{Namensliste \\ der Teilnehmer an der Expertenbefragung für den Artikel „Die Patientensicherheit in der Schweiz, Resultate einer Expertenbefragung“ \\ Schweizerische Ärztezeitung,2009;90: 48 \\ (Redaktionelle Verantwortung bei den Autoren)}

\section{Autoren}

Norbert Rose ${ }^{1}$, Maria-Anna J. Ortner ${ }^{2}$, Christa Meyenberger ${ }^{3}$ und André L. Blum ${ }^{4}$

1: Abteilung Qualitätsmanagement, Kantonsspital St.Gallen; 2: Gastroenterologie, Inselspital Bern; 3: Gastroenterologie/Hepatologie, Kantonsspital St.Gallen; 4: Faculté de Biologie et Médecine, Lausanne

\section{Korrespondenzadresse:}

Dr. med. Norbert Rose

Leiter Abteilung Qualitätsmanagement

Kantonsspital St.Gallen

$\mathrm{CH}-9007$ St.Gallen

\section{Dank}

Das Autorenteam bedankt sich bei den Experten für die Teilnahme und bei der Stiftung Patientensicherheit, für die Benutzung der Namensliste der Referenten der Tagung "Patientensicherheit Schweiz 2007".

\section{Namensliste der Teilnehmer an der Befragung}

Amstad, Basel; Bonnabry, Genf; Bregenzer, Aarau; Brückner, Basel; Frank, Zürich; Frey, Zürich; Gandon, Bern; Gruber, Zürich; Haller, Genf; Hanselmann,St.Gallen; Heim, StarrkirchWil; Henz, St.Gallen; Hochreutener, Zürich; Hofstetter, Interlaken; Kaufmann, Solothurn; Kaufmann, Basel; Kehrer, St.Gallen; Kessler, Zürich; Kessler, Luzern; Knuchel, Solothurn; Kressig, Basel; Laffer, Biel; Lange, St.Gallen; Langenegger, Bern; Langewitz, Basel; Lehmann, Freiburg; Lessing, Witten; Locher, Prilly; Manser, Zürich; Martinelli, nterlaken; Muff, Riaz; Oertle, Thun; Omega Huber, Zürich; Otto, Bern; Perneger, Genf; Perrier, Genf; Pfaff, Köln; Pougnier, Münsingen; Regli, Bern; Rhiner, Zofingen; Roggo, Bern; Röthlin, Scherzingen; Scheidegger, Basel; Schiegg, Uetikon am See; Schilling, Zürich; Schneider, Bern; Schweingruber, Bern; Schwendimann, Basel; Siegrist, St.Gallen; Staines, Clarmont; Steccanella, Bern; Steffen, St.Gallen; Suter, Genf; Teske, Solothurn; von Below, Biel; Wieser, Winterthur; Zahnd, Bern; Zeltner, Bern; Zollinger, Bern. 